\title{
LOS GOBIERNOS GESTORES AL FRENTE DEL AYUNTAMIENTO DE BARCELONA (1934-1936)
}

\author{
ALEXANDRE SOLANO \\ UNIVERSIDAD DE BARCELONA
}

RESUMEN: El periodo entre la proclamación del Estado Catalán de 1934 y las elecciones generales de 1936 se caracteriza por la sustitución de numerosos ayuntamientos, así como de la Generalitat, por comisiones gestoras gubernamentales. En el caso de Barcelona hay diferentes períodos, que conllevan cambios de concejales y hasta de alcaldes, en un consistorio donde los partidos que formaban parte de las comisiones estaban en constante disputa por el poder. Pese a durar más de un año, hay un vacío historiográfico sobre las comisiones, el talante de cada gobernante, así como las principales polémicas. El análisis de estas cuestiones nos permite profundizar más en este periodo.

PALABRAS CLAVE: Barcelona, Ayuntamiento de Barcelona, Lliga Catalana, Partido Republicano Radical.

\section{THE CARETAKER GOVERNMENTS IN CHARGE OF THE BARCELONA CITY COUNCIL (1934-1936)}

\begin{abstract}
The period between the proclamation of the Catalan State in 1934 and the general elections in 1936, is characterized by the replacement of numerous municipalities, as well as the Generalitat, by caretaker government commissions. In the case of Barcelona there are different periods, which entail changes of councillors and even mayors, in a consistory where the parties that formed part of the commissions were in constant dispute for power. Despite lasting more than a year, there is a historiographic gap on the commissions, the mood of each ruler, as well as the main controversies allows us to delve further into this period.
\end{abstract}

KEYWORDS: Barcelona, Barcelona City Council, Catalan League, Radical Republican Party. 
El 7 de octubre de 1934, el mismo día que eran detenidos los líderes de la Generalitat de Cataluña y del Ayuntamiento de Barcelona, el teniente coronel José Martínez Herrera se situaba al frente del ayuntamiento como gestor único.

Este nombramiento daría inicio a un periodo que se prolongaría hasta febrero de 1936, con gobiernos gestores, designados por los gobernantes, con muchos cambios y sin una iniciativa política importante. Este último punto provoca que haya un vacío historiográfico sobre esta cuestión, centrándose más en la reorganización de las izquierdas que en los responsables de gestionar la ciudad durante un periodo que sobrepasa el año.

Las principales fuentes de la actuación de la mayoría de izquierdas apartada del poder son las publicaciones contemporáneas, los estudios sobre ERC - como la obra de Maria Dolors Ivern -, ${ }^{1}$ así como la biografía del alcalde Carles Pi Sunyer. ${ }^{2}$ En referencia a la actuación del ayuntamiento en este periodo, la información que se tiene actualmente es muy escueta, incluso en la obra de Ferran Aisa, autor del principal texto sobre esta institución local durante la República. ${ }^{3}$ Tenemos como fuentes principales, la documentación generada por el mismo ayuntamiento, las publicaciones de la época y de forma complementaria, en estudios sobre los partidos políticos que formaron parte de las comisiones gestoras, como la Lliga Catalana, ${ }^{4}$ o biografías sobre los principales dirigentes, como la del líder regionalista Francesc Cambó ${ }^{5}$ o la del gobernador general de Cataluña y presidente del Consejo de Ministros Manuel Portela. ${ }^{6}$

En Barcelona, el periodo gestor consta de diversas etapas, con cambios de concejales y hasta de alcaldes y con unos partidos en constante disputa. Las formaciones presentes tenían dos objetivos, uno que las enfrentaba, y otro que las obligaba a colaborar. El primero era hacerse con el poder y aprovechar esta oportunidad única para mandar, en un momento en el que no existía ni control ni una oposición como tal y que gobernar dependía más de los pactos que había en Madrid que del apoyo popular; el segundo era mostrar una buena imagen conjunta, que visibilizara una alternativa en la gestión municipal a las izquierdas y que permitiese a los partidos representados en la comisión gestora de ganar las siguientes elecciones en Barcelona y Cataluña.

\footnotetext{
${ }^{1}$ IVERN I SALVÀ, Maria Dolors: “Esquerra Republicana de Catalunya (1931-1936)”, Barcelona, Edicions de l'Abadia de Montserrat, 1988.

2 PI SUNYER, Carles: “La República y la Guerra: memorias de un político catalán”, Mèxic D. F., Oasis, 1975.

3 AISA, Ferran: "L'Ajuntament de Barcelona (1931-1939): república, guerra i revolució", Barcelona, Base Històrica, 2009.

${ }^{4}$ MOLAS, Isidre: “La Lliga Catalana”., Barcelona, Edicions 62, 1972.

${ }^{5}$ CAMBÓ, Francesc: “Memòries (1876-1936)”., Barcelona, Alpha, 1981.

${ }^{6}$ MERA COSTAS, Pilar: "Monárquico, republicano, liberal. Biografía política de Manuel Portela Valladares"., Tesis doctoral, Universidad Computense de Madrid, 2016.
} 


\section{El gobierno militar del teniente coronel Martínez}

En un inicio, el ayuntamiento, ocupado militarmente, tenía como único objetivo evitar que se detuvieran los servicios municipales. La primera medida era retomar la vida económica del ayuntamiento, así que, el 9 de octubre, dos días después de la toma, se ponía en contacto con los representantes del Banco Hispano-Colonial, ya que desde el sábado anterior las operaciones económicas habían sido paralizadas y estaban próximas al colapso.

Más allá de esta cuestión no existía ninguna instrucción más, sólo mantener el ayuntamiento con el asesoramiento de altos funcionarios, y con la colaboración de los concejales de la Lliga Catalana y los radicales, a pesar de que habían sido cesados del cargo. En el ayuntamiento no existían ni las sesiones de pleno ni de consejo ni acuerdos a anunciar. El siguiente número de la Gaceta Municipal no sería hasta el 7 de enero de 1935 y el único horario público era el establecido para las visitas, los martes y viernes de $10.00 \mathrm{a} 11.00 \mathrm{~h}^{7}$

El 12 de octubre el teniente coronel Martínez Herrera establecía que, en estado de guerra y con las funciones en manos de la autoridad militar, los decretos de alcaldía tuvieran el mismo valor que los acuerdos adoptados en sesión plenaria, e incluso que se dejara sin efecto, o suspendidos, cualquier reglamento que pudiera oponerse y contradijera las medidas, fueran sobre servicios municipales, expedientes a funcionarios, sanciones o nombramientos. ${ }^{8}$

Para que no se detuviera el funcionamiento de los servicios, se daba a los jefes administrativos de Sección y al jefe del Departamento de Circulación, la autorización para resolver y firmar las comunicaciones en todos los asuntos de carácter reglamentario, solo excluyendo los que supusieran un pago por parte del ayuntamiento, que pasaban por manos del gestor único.

\section{La política de control de Martínez Herrera}

El teniente coronel Martínez Herrera, a pesar de ejercer el cargo por una ocupación militar, no mostró una actitud extremadamente revanchista. El día 9 de octubre ya se decía a los reporteros que se avisara de la inutilidad de las delaciones y ofrecimientos de documentación comprometedora, porque no tenía atribuciones, y que las denuncias tenían que ir a la autoridad judicial. ${ }^{10}$

\footnotetext{
${ }^{7}$ Archivo Histórico de la Ciudad de Barcelona (AHCB). La Veu de Catalunya, 23-11-1934, Ed. Mañana, p. 3. Barcelona.

8 Archivo Municipal Contemporáneo de Barcelona (AMCB). Ayuntamiento de Barcelona. GM-279. Actuaciones practicadas durante la alcaldía del Sr. Teniente Coronel Martínez Herrera.

9 AMCB. Ayuntamiento de Barcelona. GM-276. Expediente General.

${ }^{10}$ AHCB. La Publicitat, 10-10-1934, p. 3. Barcelona.
} 
También hay que señalar que el teniente coronel enviaba al Presidente de la República una carta para que "baciéndome eco del sentimiento general de la Ciudady del Ayuntamiento eleva V.E. la petición interesando gracia de indulto para el Mando Pérez. Farràs y Capitán Escofet, en caso de que la Superioridad confirme la sentencia de muerte dictada" por el Consejo de Guerra, ${ }^{11}$ así como al teniente coronel Joan Ricart. ${ }^{12}$

Sin embargo, en un inicio las publicaciones vinculadas a los partidos catalanistas de izquierdas, como 'La Humanitat', 'L’Opinió' y 'La Publicitat', entre otras publicaciones, eran suspendidas indefinidamente y a pesar de la situación, el teniente coronel Martínez Herrera se quejó de la tibieza de la censura en algunos casos. ${ }^{13}$

En la Fiesta de la Raza (12 de octubre) se consultaba el caso de las banderas, en la que el general Batet ordenaba poner la republicana y la catalana, pero cambiando el orden. Arriba del edificio la de la República y, en el balcón grande, la señera. ${ }^{14}$

El oficial del ejército también dedicó gran parte de las actuaciones a resolver problemas que podríamos definir como 'cuestiones militares' en la ciudad. El 9 de octubre hacía un decreto de máxima urgencia, seguramente porque desconocía cuánto tiempo estaría al frente del ayuntamiento, para hacer un proyecto de alumbrado en las calles que circundaban los cuarteles de la calle Sicilia, "para ser indispensable encontrándose en la zona los cuarteles indicadas". "También daba 5.000 pesetas a la suscripción abierta en favor de las víctimas del ejército con motivo de los sucesos del 6 de octubre, se concedía con un decreto 3.000 pesetas por la suscripción abierta para el 'Patronat de Viudes i Òrfenes de Militars i Marins', así como 7.713,63 pesetas a la casa' Hijos de Domingo Batet'. ${ }^{16}$

Hay que sumar la cesión del Palacio de Bellas Artes al Comité Impulsor de Compenetración Hispano-Americana, que enviaba una carta al alcalde accidental que "por tratarse de un acto españolista, el gobierno de la Generalitat de Cataluña [...] suspendía la celebración de este acto. Libre ya Cataluña de dominadores - de ideales separatistas, es momento que los que sienten un amor a la Patria Española, puedan manifestarse", pidiendo la cesión del Palacio de Bellas Artes, la cesión de la Banda Municipal y el engalanamiento del palacio de forma solemne, además de decidir que el dinero de las taquillas iría destinado a ayudar a las familias de soldados o guardias civiles participantes en los hechos de octubre. La respuesta fue acceder a todas las peticiones, cediendo el Palacio de Bellas Artes el 4 de noviembre, enviando la Banda Municipal y ordenando a los técnicos municipales que fuera engalanado. ${ }^{17}$

11 AMCB. Ayuntamiento de Barcelona. GM-276.

12 Ibidem.

13 AMCB. Ayuntamiento de Barcelona. GM-279.

${ }^{14}$ La Veu de Catalunya, 11-10-1934, Ed. Mañana, p. 8.

15 AMCB. Ayuntamiento de Barcelona. GM-276.

${ }^{16}$ Ibidem.

${ }^{17}$ Ibidem. 


\section{Guerra por la alcaldía}

El teniente coronel avisaba el 18 de octubre que no parecía probable que de manera inmediata hubiera un cambio en la organización de la vida municipal barcelonesa, aunque afirmaba, "ustedes saben cómo deseo dejar estos asuntos, y lamento que no sea de manera inmediata" y ya se vislumbraba que la salida pasaría por una comisión gestora, con un alcalde del Partido Republicano Radical (PRR). ${ }^{18}$

El gobierno de España estaba encabezado por Alejandro Lerroux y contaba con ministros del PRR, la Confederación Española de Derechas Autónomas (CEDA), el Partido Republicano Liberal Demócrata y el Partido Agrario Español. De estos, solo los radicales tenían una estructuración importante en Barcelona y una experiencia sólida en el gobierno municipal. ${ }^{19}$

El diario 'La Publicitat', el 9 de noviembre, ya señalaba que posiblemente el jefe de los radicales en Cataluña, Joan Pich i Pon, sería nombrado alcalde de Barcelona. El aspirante, junto con el radical Alfred Sedó, que también estaba entre las opciones, se reunían el día 7 con el subsecretario de Gobernación, Eduardo Benzo y, al día siguiente, Pich se reunía con el general Batet. ${ }^{20}$ Incluso se apuntaba como a aspirante a Emiliano Iglesias, diputado por Pontevedra, que el 26 de noviembre era recibido efusivamente en la administración de arbitrios, y se relacionaba este hecho con la voluntad de intervenir en el ayuntamiento antes de que lo hiciera Pich i Pon. ${ }^{21}$ La rivalidad de Iglesias-Pich por el predominio lerrouxista de Barcelona era presente desde los años veinte. ${ }^{22}$ Por su parte, Martínez Herrera afirmaba que desconocía todo lo que hacía referencia a la reconstitución del ayuntamiento. ${ }^{23}$

Los radicales habían aprovechado la destitución de la mayoría de los cargos electos para nombrar en estos puestos a gente afín a la formación. El Consejo Regional del Partido Radical recordaba a los comités provinciales del partido la necesidad de recoger, con urgencia, de los comités de ciudades y pueblos, las listas con los nombres de los militantes que pudieran ocupar plazas en comisiones gestoras, secretarios municipales y regentes de escuelas de primera enseñanza entre otros, por "la reorganización política y administrativa de la región catalana". ${ }^{24}$

\footnotetext{
${ }^{18}$ La Veu de Catalunya, 18-10-1934, Ed. Mañana, p. 10.

19 BALCELLS, Albert, CULLA, Joan B., y MIR, Conxita: "Las eleccions generals a Catalunya de 1901 a 1923 ". Barcelona, Fundació Jaume Bofill, 1982, p. 224.

20 El Mirador, 9-11-1934, p. 1. Barcelona., Ateneo Barcelonés

${ }^{21}$ La Veu de Catalunya, 28-11-1934, Ed. Mañana, p. 8.

22 La Veu de Catalunya, 28-11-1934, Ed. Mañana, p. 8.

${ }^{23}$ La Veu de Catalunya, 13-11-1934, Ed. Mañana, p. 11.

${ }^{24} \mathrm{La}$ Veu de Catalunya, 4-12-1934, Ed. Mañana, p. 1.
} 


\section{La llegada de Pich i Pon como gestor único}

El 11 de enero, un día después de que Manuel Portela ya hubiera sido nombrado gobernador general de Cataluña, el jefe regional de los radicales, Joan Pich i Pon, recibía la vara de alcalde en el Saló de Cent de parte de Martínez Herrera, con presencia de ex concejales y presidentes de centros radicales. Pich i Pon asumía las facultades otorgadas por el estado de guerra, o sea que, a nivel legal, simplemente suponía el cese de Martínez Herrera y el nombramiento de Pich. ${ }^{25}$

Como remarcaba 'La Veu de Catalunya', había "un movimiento extraordinario que contrastaba con el silencio y la quietud de los días de la alcaldía marcial. Habian desaparecido los militares $y$, en cambio, estaba lleno de radicales". ${ }^{26}$ Entre otros cargos, el nuevo alcalde nombraba como secretario particular a Joan Pich Salarich, su sobrino. ${ }^{27}$

El objetivo del alcalde era comenzar las gestiones para hacer una comisión gestora, y aseguraba que la CEDA, pese a no haber tenido representación en las elecciones municipales, debía tener presencia porque era el partido más votado en España y sería perjudicial para Barcelona y Cataluña no tener la colaboración del partido. ${ }^{28}$

La derecha de ámbito estatal había respetado el campo de acción de la Lliga en Cataluña, por la similitud de sus posiciones. ${ }^{29}$ Sin embargo, aprovechando el debate sobre la ley de contratos de conreo, en el cual muchos propietarios consideraban que los regionalistas tenían una posición demasiada moderada, se organizó Acció Popular Catalana, formación adherida a la CEDA que seria constituida justo en octubre de $1934 .^{30}$

\section{La obra de Pich i Pon como gestor único}

El alcalde accidental, Joan Pich i Pon, a diferencia de Martínez Herrera, anunció proyectos que no correspondían a su interinidad. Por ejemplo, en la comisión de Ensanche, se reunían, a falta de concejales, los vocales propietarios designados por la Cámara Oficial de la Propiedad, de la cual, hasta el nombramiento como alcalde, Pich i Pon era el presidente. ${ }^{31}$ Por lo tanto, solo había unos representantes que defendían unos intereses y una clase. En el

\footnotetext{
25 AMCB. Ayuntamiento de Barcelona. GM-281/1. Alcaldía del Sr. Pich.

${ }^{26}$ La Veu de Catalunya, 13-1-1935, Ed. Mañana, p. 1.

${ }^{27}$ La Publicitat, 13-1-1935, p. 2.

${ }^{28}$ La Publicitat, 12-1-1935, p.1.

${ }^{29}$ RAGUER, Hilari: "La Unió Democràtica de Catalunya i el seu temps (1931-1939)"., Montserrat (Barcelona), Publicacions de l'Abadia de Montserrat, 1976, p. 213.

${ }^{30}$ MOLAS, Isidre, 1972, op. cit., p. 258.

31 La Publicitat, 6-2-1935, p. 2.
} 
mandato de Martínez Herrera, el alcalde y los más grandes de entre los propietarios se reunían despachando los asuntos de trámite, mientras los permisos y otros asuntos eran pasados a la Junta de Jefes. ${ }^{32}$ Pero con Pich i Pon, había una clara voluntad de desarrollar una obra.

El alcalde Pich anunciaba un programa de obras y reformas, y el 26 de enero inauguraba el Mercado del Ninot, mercado que ya se había inaugurado en 1933 cuando se pusieron en servicio las primeras naves. ${ }^{33}$ Entre los proyectos había diversas reformas, y Pich i Pon aseguraba que reuniría entidades patronales, especialmente de la construcción, jefes letrados, técnicos y representantes del Banco Hispano Colonial, para estudiar una fórmula para evitar que los dos millones de pesetas que costaba la apertura de la calle Fortuny debieran ser pagados totalmente como mejoras por los propietarios, porque sería una carga demasiado grande para ellos. ${ }^{34}$ Por lo tanto, había un claro sesgo en defensa de los intereses de los propietarios, a pesar de tener que defender los intereses generales de la ciudad.

Hay que señalar una política a favor del partido. El 30 de abril de 1935, por ejemplo, un decreto del alcalde daba el Casino Republicano Radical de la Barceloneta (de su partido) una subvención de 800 pesetas por parte del consistorio en beneficio de un joven mutilado. ${ }^{35}$ También es de remarcar que en la fiesta de Carnaval que celebró Pich i Pon, mientras los años anteriores había costado 25.000 pesetas, el gestor único se gastó 100.000 pesetas en un banquete muy fastuoso. Por lo tanto, Pich i Pon a pesar de ser gestor, utilizó en gran parte su posición en el ayuntamiento para dar una orientación concreta y con la defensa clara de ciertos intereses de clase y de partido.

\section{La posición contraria de la Lliga}

La Lliga Catalana se opuso en un primer momento a la alcaldía del radical. El diputado Joan Ventosa i Calvell, denunciaba que, en las elecciones de 1934, las izquierdas habían obtenido 162.000 votos, la Lliga 132.000 y los radicales 21.000; pero en cambio, los diez concejales de la Lliga habían sido destituidos y de nada servía que les amparara la ley. El artículo 56 de la ley de Orden Público decía que no serían separados de sus cargos los individuos que continuaran en el uso de sus facultades civiles. ${ }^{36}$ En cambio, a los radicales se les daba la alcaldía.

\footnotetext{
32 La Publicitat, 8-2-1935, p. 3.

${ }^{33}$ La Publicitat, 27-1-1935, p. 3.

${ }^{34}$ La Humanitat, 2-2-1935, p. 8. Barcelona. Universidad de Barcelona. CRAI Biblioteca del Pabellón de la República.

${ }^{35}$ AMCB. Ayuntamiento de Barcelona. GM-281/1.

${ }^{36}$ La Humanitat, 7-2-1935, p. 1.
} 
Como consecuencia decidían no dar ningún nombre para una posible comisión gestora. ${ }^{37}$ Cabe señalar, además, que el vicepresidente del Parlamento de Cataluña, Martínez Domingo, denunciaba la ley del 2 de enero de 1935, porque suspendía el Parlamento, a pesar de no haber actuado en los hechos de octubre. El partido, pese a estar en contra de la proclamación del Estado Catalán de 1934, mostraba una actitud de defensa del autogobierno y focalizaba la crítica en la actuación de los dirigentes de ERC.

El 20 de enero, 'La Veu de Catalunya' - el órgano de la Lliga Catalana - publicaba una carta abierta a Joan Pich i Pon de Xavier Calderó, concejal de la ciudad y vicepresidente de la asamblea del Ayuntamiento, en la que afirmaba que se había enterado por la prensa de actuaciones como la prohibición de apertura de nuevos establecimientos de venta de carne, el propósito de derribar las Atarazanas o la apertura de la calle Fortuny y de la Gran Vía C que debía unir la Rambla con la Via Laietana -, y denunciaba que aunque Pich había dicho que no era legalista, "hay un principio de derecho universalmente admitido, como fase fundamental de toda organización civilizada, que dice que no es lícito ignorar las leyes y mucho menos alegar, como excusa para incumplirlas o vulnerarlas, aquella ignorancia 'y señalaba que 'quiere prescindir VS de toda asistencia porparte de los legitimos representantes de la ciudad” y “que un señor Gestor lleva a ejecución determinaciones que son de incumbencia de las comisiones permanentes, unas, y del Ayuntamiento en Pleno, otras".

En referencia a decisiones como la reposición de Manuel Ribé al frente de la Guardia Urbana, y la reorganización y el desarme del cuerpo, señalaba que no estaba en contra, pero que las disposiciones eran ilegales, y que escapaban de las facultades del alcalde gestor y justamente remarcaba que en un acuerdo del Pleno se había acordado que todos los "nombramientos, traslados, ascensos y destituciones, realizados en el Cuerpo de la Policía de Circulación y Urbana debian ser objeto de un estudio a fondo por los jefes letrados del Ayuntamiento, los que deberian emitir dictamen sobre cada caso, respecto a su legalidad'.38

En el mismo sentido, el que era jefe de la minoría de la Lliga en el municipio, Lluís Duran i Ventosa, dejaba claro que, "mi criterio es que se debe cumplir la ley. Alli donde no llegue, sus deficiencias no pueden ser llenadas más que mediante reglas generales. Nunca una aplicación de criterios arbitrarios para cada caso, procurando cada partido sacar el mejor provecho de una situación crítica". ${ }^{39}$

En la actuación de la Lliga Catalana había una crítica a la vulneración de la legalidad, pero en gran parte estaba la denuncia del poder que se había dado a los radicales, pese a tener un apoyo mucho menor. En el fondo había una demanda de restablecer el ayuntamiento popular, menos los concejales procesados, que diera una mayoría regionalista, un poder

${ }^{37}$ La Veu de Catalunya, 18-1-1935, Ed. Mañana, p. 10.

${ }^{38}$ La Veu de Catalunya, 20-1-1935, Ed. Mañana, p. 11.

${ }^{39} \mathrm{La}$ Veu de Catalunya, 22-1-1935, Ed. Mañana, p. 11. 
mayor en la comisión o simplemente elecciones, que con el fracaso revolucionario de ERC y la situación que se había producido posteriormente, era de prever una mayoría de la Lliga si se hacían elecciones inmediatamente.

En el Congreso de los Diputados, Ventosa i Calvell presentaba una proposición de ley en la que se pedía el restablecimiento de la vida municipal en Cataluña, que recibía el voto en contra de la mayoría de gobierno (radicales, los cedistas, los agrarios y los melquiadistas), mientras las otras minorías transversalmente (izquierdas, progresistas, mauristas, monárquicos, tradicionalistas, vascos, federales y elementos dispersos como el conde Romanones) votaban a favor. ${ }^{40}$

Finalmente, decían que la condición indispensable para colaborar con el régimen era mantener los concejales contra los que no había ningún cargo y proveer las vacantes, en primer término, con los suplentes que fueron elegidos. ${ }^{41}$ Según Antoni Rovira i Virgili en 'La Humanitat', Francesc Cambó había puesto como condición que el método fuera automático, porque si era a dedo, la gran mayoría sería afín a quien hiciera los nombramientos, por lo tanto, aceptaba cualquier fórmula siempre que fuera automática. Incluso Cambó mencionaba algunas posibilidades: los exconcejales del 12 de abril de 1931, los primeros o últimos contribuyentes, representantes de los sindicatos, entre otras opciones. ${ }^{42}$

\section{Hacia la creación de las comisiones gestoras}

La creación de las comisiones comportaba problemas legales; según el diario 'El Debate' las nuevas autoridades en Barcelona estaban perplejas, ya que la suspensión del Parlamento no alteraba la vigencia de las leyes, entre ellas la Ley Municipal, que prohibía terminantemente la constitución de comisiones gestoras. Solo parecía posible por la Ley de Orden Público, ya que mientras había el estado de guerra, había un gestor único como delegado del general de división; primero había sido el teniente coronel Martínez Herrera y posteriormente Pich i Pon. ${ }^{43}$

La ley del 2 de enero establecía el régimen transitorio de Cataluña, en la que daba al gobernador general las funciones de presidente y del Consejo Ejecutivo de la Generalitat,

\footnotetext{
40 La Publicitat, 8-2-1935, p. 2.

${ }^{41}$ La Veu de Catalunya, 22-2-1935, Ed. Mañana, p. 15.

${ }^{42}$ La Humanitat, 7-5-1935, p. 12.

${ }^{43}$ La Humanitat, 19-1-1935, p. 7.
} 
pero no le daba la facultad de escoger el alcalde de Barcelona. Por lo tanto, Pich i Pon tenía las mismas atribuciones que Martínez Herrera, pero pasando de un alcalde de carácter militar a uno de carácter civil. ${ }^{44}$

Después de prolongarse el tiempo de constitución de las gestoras por las dificultades de pactar con el resto de fuerzas, el 22 de enero se iniciaba una gestora de jefes de sección del ayuntamiento, que debía reunirse y examinar los asuntos de varios departamentos para proponer al alcalde un conjunto de soluciones que consideraran convenientes "porque era imposible que las cosas continuaran como hasta ahora". ${ }^{45}$

El 25 de febrero, el Consejo de Ministros aprobaba un decreto que facultaba al Gobernador General de Cataluña la capacidad de destituir ayuntamientos y nombrar Comisiones gestoras, a pesar de contradecir lo que decía el régimen local desarrollado por el Estatuto. $^{46}$

Cuanto más tiempo pasaba, más se incrementaban las críticas, 'El Diluvio' publicaba una nota señalando que "transcurren las semanas y se regulariza la puesta en marcha de la administración municipal. A principios de la semana anterior vino indiciar el señor Pich que con mucha brevedad quedarian designados los gestores que junto con él debian regir los destinos del municipio barcelonesa", "los asuntos municipales están casi todos en un verdadero atasco. Servicios importantísimos se encuentran en el más completo abandono". 47

El 3 de abril, Manuel Portela, al ser nombrado ministro de Gobernación, dejaba el cargo de gobernador civil, siendo nombrado Pich i Pon, que se convertía en Presidente de la Generalitat, Gobernador General y Alcalde de Barcelona. El 14 de abril eran devueltos los traspasos a la Generalitat, pero se retenía el orden público.

Una de las primeras medidas, ya como gobernador general interino, era ordenar la destitución de todos los jueces municipales de Cataluña, más de mil. La Generalitat de Cataluña ya había decretado una renovación extraordinaria, hecha por la Audiencia de Barcelona, casi sin modificaciones, pero en esta ocasión el presidente del Consejo de Justicia Municipal denunciaba que "la disposición de Pich $i$ Pon es un abuso de Poder". Uno de los principales motivos de suspensión, que afectó a doscientos jueces, era por haber tomado parte "en el movimiento subversivo". ${ }^{48}$

\footnotetext{
${ }^{44}$ La Veu de Catalunya, 12-1-1935, Ed. Mañana, p. 11.

${ }^{45}$ La Publicitat, 23-1-1935, p. 3.

${ }^{46}$ La Humanitat, 27-5-1935, p. 3.

${ }^{47}$ La Humanitat, 26-2-1935, p. 8.

${ }^{48}$ La Veu de Catalunya, 10-4-1935, Ed. Mañana, p. 11.
} 


\section{Las Comisiones Gestoras}

El 28 de abril, después de siete meses durante los cuales el ayuntamiento de Barcelona estuvo formado solamente por un único gestor, Pich i Pon, como gobernador interino, firmaba un decreto para regular y normalizar los organismos municipales.

Entre otras medidas, se ampliaba la configuración que inicialmente tenía que tener la comisión. La ampliación era un movimiento para contentar a la Lliga, que quedaba en minoría, pero a cambio se mantenía los concejales regionalistas elegidos en las elecciones. 'La Veu de Catalunya' titulaba su editorial como "Cap a la normalitat de la vida municipal” (Hacia la normalidad de la vida municipal), donde consideraba el decreto del Gobierno General interino como el inicio del retorno a la normalidad de la vida municipal catalana, y el decreto, como una confirmación del régimen autonómico de Cataluña. ${ }^{49} \mathrm{El}$ discurso iba en la línea de colaborar con el resto de fuerzas de centroderecha, y a la vez, justificar la participación en un régimen temporal que había substituido a los representantes elegidos democráticamente.

La Lliga Catalana conseguía que se reintegrasen sus concejales, pero la elección de los concejales no era automática, por lo que, por las izquierdas era considerado como una victoria de Pich i Pon, que maniobraba para asegurar una mayoría a sus postulados. La lista de concejales hecha pública era la siguiente ${ }^{50}$ :

\section{Cuadro 1}

\section{Lista de la propuesta de concejales de la primera comisión gestora}

\begin{tabular}{|c|c|c|c|}
\hline \multicolumn{2}{|c|}{ Comisión Gestora } & \multicolumn{2}{|c|}{ Han declinado, pese a poder formar parte } \\
\hline \multirow[t]{11}{*}{10 regionalistas } & Josep M. Blanch Romeu & \multirow[t]{11}{*}{ ERC } & Jaume Serra Húnter \\
\hline & Andreu Bausili Sanromà & & Josep $\mathrm{M}^{\mathrm{a}}$ Massip Izàbal \\
\hline & Xavier Calderó Corones & & Ramon Boter Mauri \\
\hline & Josep Codolà Gualdo & & Ramon Eroles Surribes \\
\hline & Lluís Duran i Ventosa & & Francesc Ferrer Ple \\
\hline & Octavi Saltor Soler & & Antoni Lloret Fumanal \\
\hline & Frederic Roda Ventura & & Gaietà Rahola Escofet \\
\hline & Ferran Sagarra & & Ramon Palomes Tudó \\
\hline & Castellarnau & & \\
\hline & Joan Soler Janer & & Delmir Ibàñez \\
\hline & $\begin{array}{l}\text { Francesc A. Vendrell } \\
\text { Tiana }\end{array}$ & & \\
\hline & a... & & \\
\hline
\end{tabular}

${ }^{49}$ La Veu de Catalunya, 30-4-1935, Ed. Mañana, p. 1.

${ }^{50}$ La Veu de Catalunya, 30-4-1935, Ed. Mañana, p. 17. 


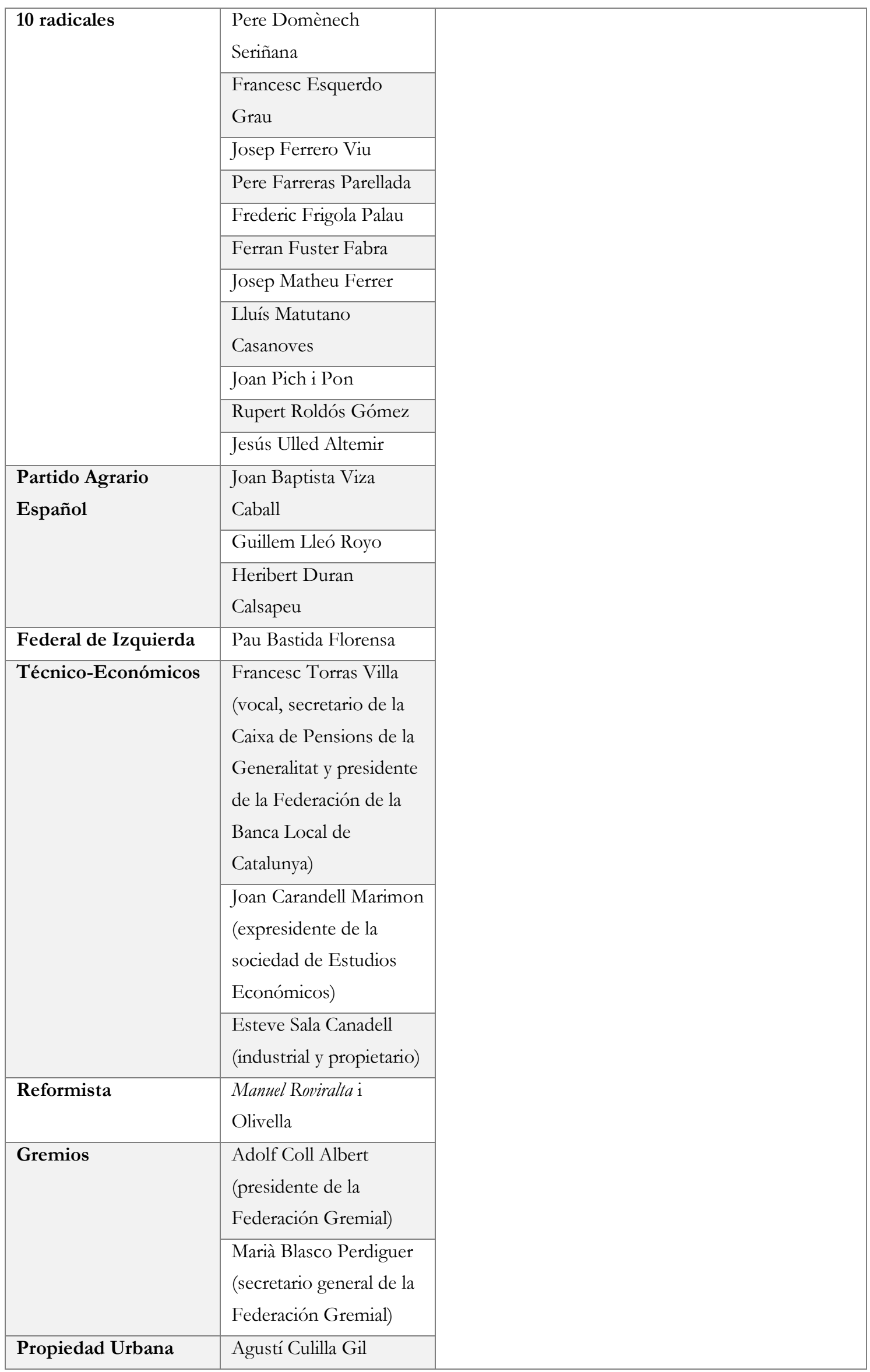

Fuente: Elaboración propia 
La lista se hacía pública sin haber sido nombrados los ochos concejales de la CEDA, que finalmente serian Francesc de Sales Jaumar de Bofarull, Cèsar Martinell Bruna, Francesc Santacana Romeu, Jordi de Camps de Casanoves, Albert de Quintana Vergés, Lluís Bassols de Climent, Antoni Miseracs y Francesc Tiffon Vila.

Por parte de la Lliga Catalana, Duran i Ventosa, que ocupaba el cargo gestor a la Generalitat era sustituido por Ramon Coll i Rodés ${ }^{51}$, mientras que Esteve Sala, presidente del FC Barcelona, rechazaba públicamente su nombramiento, ${ }^{52}$ motivo por lo cual era substituido por Luis Gonzaga Maria Cascante i Portabella, ingeniero industrial de la CEDA. ${ }^{53}$

Los concejales de izquierdas no suspendidos, Josep Maria Massip, Jaume Serra Húnter y Ferran Boter, porque no pudieron estar presentes en la votación de apoyo a la proclamación del Estado catalán de 1934, así como los suplentes, hacían llegar un documento al Departamento de la Presidencia de la Generalitat, donde renunciaban a formar parte de las comisiones gestoras, ya que "no pueden reconocer validę legal a las disposiciones en virtud de las cuales se ban hecho los mencionados nombramientos" porque "infringe preceptos legales de cumplimiento inexcusable para los que quieran representar la autoridad". ${ }^{54}$

Cabe señalar que el alcalde gestor Pich i Pon, enviaba, el 25 de abril, una carta a Carles Pi Sunyer, "como elemento de Esquerra Republicana de Cataluña al que, por el cargo ostentado en el ayuntamiento, se puede estimar con condición de jefe de dichos elementos políticos", sobre si los concejales no suspendidos aceptaban o renunciaban, pidiendo una respuesta urgente. La respuesta de Pi Sunyer, alcalde popular de Barcelona hasta su destitución el 7 de octubre, no se hacía esperar. El mismo 25 de abril respondía que los miembros de la ejecutiva del partido que estaban en Barcelona habían decidido que "no podía considerarse legalmente constituidos los Ayuntamientos" porque no se basaban en el resultado de las elecciones municipales del 14 de enero de 1934 y que en caso de que alguien aceptara formar parte de ellas, no podría hacerlo en nombre y representación de ERC en ningún ayuntamiento de Cataluña. Ningún concejal iba a buscar ninguna credencial, ni se presentaba a la sesión previa a la constitución. ${ }^{55}$

En la sesión de constitución, que se producía el 6 de mayo, Xavier Calderó era elegido presidente de la Asamblea y se repartían las tenencias aún sin los cedistas, a quienes se les reservaba la primera y la séptima tenencia de alcaldía:

\footnotetext{
51 AMCB. Ayuntamiento de Barcelona. GM-281/2 Alcaldía: Expediente de los alcaldes sres. Pich, Jaumar y Ulled.

52 Ibidem.

53 Ibidem.

${ }^{54}$ La Publicitat, 8-5-1935, p. 6.

55 AMCB. Ayuntamiento de Barcelona. GM-281/2.
} 


\section{Cuadro 2}

Cargos de la primera comisión gestora

\begin{tabular}{|l|l|}
\hline Alcalde & Joan Pich i Pon \\
\hline Primer Consejero - Concejal & En blanco (designado posteriormente Francesc Jaumar) \\
\hline Segundo Consejero- Concejal & Jesús Ulled \\
\hline Tercer Consejero- Concejal & Ramon Coll i Rodés \\
\hline Cuarto Consejero- Concejal & Joan Baptista Viza \\
\hline Quinto Consejero- Concejal & Josep Codolà \\
\hline Sexto Consejero- Concejal & Josep Farrero \\
\hline Séptimo Consejero- Concejal & Andreu Bausili \\
\hline Octavo Consejero- Concejal & En blanco (designado posteriormente Francesc Santacana) \\
\hline Presidente de la Asamblea & Xavier Calderó \\
\hline Vicepresidente primero & Francesc Torras \\
\hline Vicepresidente segundo & Frederic Frigola \\
\hline
\end{tabular}

Fuente: Elaboración propia

En los discursos hechos por los diferentes grupos municipales, se demostraba que en ningún caso iban al ayuntamiento simplemente a gestionar, sino a que iban a hacer política. Joan Baptista Viza, líder de los agrarios, afirmaba que venía a defender los derechos "de los propietarios naturales de la tierra" y anunciaba la tarea de rehacer la tradición confesional. Por su parte, la Lliga Catalana hablaba de restitución y no de Comisiones Gestoras, y que tan solo unos concejales habían sido apartados a consecuencia de los hechos del 6 de octubre. ${ }^{56}$

El concejal Joan Soler Janer, tradicionalista, escogido dentro de la candidatura municipal de la Lliga, anunciaba que actuaba como una minoría unipersonal desligada de cualquier otro partido; también formaba una minoría unipersonal el reformista Manuel Roviralta, mientras que Francesc Torras, exdiputado provisional de la Dictadura, era portavoz de los económicos y de Pau Bastida. ${ }^{57}$ Después de esta primera sesión, se incorporaban los concejales de la CEDA. Su líder, Francesc Jaumar, obtenía la primera tenencia, mientras Francesc Santacana Romeu, la octava. La publicación satírica 'l’Esquella de la Torratxa', para remarcar el carácter 'de clase' del nuevo consistorio, señalaba el gran número de apellidos con "de": Francesc Jaumar de Bofarull; Jordi de Camps de Casanoves; Lluís Bassols de Climent; Ferran de Sagarra de Castellarnau i Albert de Quintana Vergès. ${ }^{58}$

\footnotetext{
${ }^{56}$ La Veu de Catalunya, 7-5-1935, Ed. Mañana, p. 21.

${ }^{57}$ La Humanitat, 7-5-1935, p. 7.

${ }^{58}$ L’Esquella de la Torratxa, 26-7-1935, p. 4., Barcelona., Ateneo Barcelonés
} 
El 15 de mayo se reunía por primera vez la Comisión de Gobierno Municipal, que acordaba que los plenos se celebrarían cada tres meses, plazo muy inferior a las sesiones mensuales del ayuntamiento popular. El cartapacio y, por tanto, los concejales que formaban parte de esta comisión gestora eran los siguientes:

\section{Cuadro 3}

\section{Lista de la primera Comisión de Gobierno Municipal}

\begin{tabular}{|l|l|l|}
\hline Nombre & Área & Partido político \\
\hline Joan Pich i Pon & Presidente & Partido Republicano Radical \\
\hline Jesús Ulled & Ensanche & Partido Republicano Radical \\
\hline Josep Farrero & Circulación & Partido Republicano Radical \\
\hline Josep Codolà & Cultura & Lliga Catalana \\
\hline Ramon Coll i Rodés & Fomento & Lliga Catalana \\
\hline Andreu Bausili & Política Social & Lliga Catalana \\
\hline Joan Baptista Viza & Finanzas & Partido Agrario Español \\
\hline Francesc Jaumar & Gobernación & CEDA \\
\hline Francesc Santacana & Provisiones & CEDA \\
\hline
\end{tabular}

Fuente: Elaboración propia

\section{La actuación de la comisión gestora}

Los diferentes partidos se repartieron las consejerías, pero no existía una orientación común. La mayoría de las formaciones (cedistas, agrarios, reformistas, etc.) difícilmente podrían obtener concejales por métodos democráticos en un sistema mayoritario como el que había y, aprovechando esta oportunidad, cada formación intentaba marcar el mejor perfil de cara a unas futuras elecciones.

La situación, sin una orientación política clara, llevaba a políticas concretas en favor de ciertos grupos representativos de potenciales votantes. El consejero municipal de gobernación Jaumar de Bofarull, líder de la minoría de la CEDA, iniciaba el mandato con un gesto a favor de los propietarios, condonando todas las multas pendientes de pago a los propietarios de fincas que habían sido sancionados por las inspecciones sanitarias. ${ }^{59} \mathrm{La}$ Comisión de Gobierno también pagó 15.000 pesetas para arreglar el órgano de la Catedral, ${ }^{60}$ un pago, que, si se entiende que se hacía a la iglesia, sería inconstitucional.

\footnotetext{
${ }^{59}$ La Humanitat, 30-5-1935, p. 9.

${ }^{60}$ La Publicitat, 1-6-1935, p. 5.
} 
En referencia a los presupuestos, el gobierno gestor prorrogaba los de abril de 1934. Para 'La Humanitat' el intento de presupuesto de Pich i Pon, con Bausili y Viza había fracasado porque las diferentes minorías no habían podido ponerse de acuerdo ni en el aumento de sueldo de los altos funcionarios ni en el número de plazas a crear para cubrir las necesidades políticas de los partidos. ${ }^{61}$

El alcalde accidental, Pich i Pon, sin embargo, prometía una obra de gobierno conjunta, como la reforma para hacer una calle que uniera la Rambla con la Via Laietana, con un presupuesto extraordinario, en el que proyectaba que fueran expropiadas más de setenta casas; además de prometer 35 millones para un Matadero nuevo. Por tanto, a pesar de no llegar a producirse los planes, la voluntad era, a pesar de ser gestor, hacer obras importantes e irreversibles para cuando ya hubieran entrado dirigentes elegidos democráticamente. $^{.2}$

En materia de personal, durante el periodo gestor se hicieron 383 ascensos o nombramientos, de los cuales, solo uno fue proveído por concurso. ${ }^{63}$ En conjunto, en 1935 había 6.115 trabajadores en el ayuntamiento. ${ }^{64}$

\section{Los enfrentamientos en el seno de las comisiones gestoras}

Pese a querer mostrar una imagen de gobierno municipal responsable, el 11 de junio, tenía lugar una de las primeras peleas públicas dentro del ayuntamiento, entre la Lliga Catalana y la CEDA, que, a pesar de compartir gobierno, se disputarían en un futuro el voto de derechas en Cataluña.

En la Comisión de Gobernación, presidida por Jaumar, con Roda y Ventura como representante de la Lliga, se acordó hacer una "ponencia para proceder a una reorganización del personaly también a una revisión del personal; sin embargo, solo unos días más tarde, Jaumar hacia una nota sobre esta cuestión, afirmando que la Comisión de Gobierno había aprobado una serie de disposiciones sobre control de personal, sin consultar a los miembros de la Comisión de Gobernación.

El suceso proseguía con unas declaraciones de Roda i Ventura, asegurando que la nota "representaba toda una desconsideración para la comisión de gobernación", ya que los miembros de están comisión habían conocido los planes por la prensa. ${ }^{65}$ Por su parte, Jaumar explicaba

\footnotetext{
${ }^{61}$ La Humanitat, 25-8-1935, p. 7.

62 La Humanitat, 25-8-1935, p. 7.

${ }^{63}$ AMCB. Ayuntamiento de Barcelona. GM-781. Documentos referentes a personal de la Asociación de obreros y empleados municipales.

${ }_{64}$ AMCB. Ayuntamiento de Barcelona. GM-121. Reforma de los servicios técnicos municipales.

${ }^{65}$ La Publicitat, 12-7-1935, p. 1.
} 
en una nota que había dos comisiones, una de ellas, nombrada por la comisión de Gobernación, para hacer un examen completo del personal nombrado antirreglamentariamente, ${ }^{66}$ y la otra, nombrada por la Comisión de Gobierno sobre las medidas de control del personal, que son las que se habían presentado.

En la reunión de la Comisión de Gobernación, Roda i Ventura presentaba una moción de censura contra el presidente Jaumar, pero los radicales, a pesar de estar molestos, para no crear problemas en la política estatal (Pacto de Salamanca) votaban en contra, igual que las otras minorías, vinculadas en mayor o menor medida a Pich i Pon, como Colilla (representante de la Cámara de la Propiedad) o Blasco (gremial pero personalmente radical). ${ }^{67}$ 'La Publicitat' afirmaba que la ruptura ya venía de lejos, de una votación en el Patronato Escolar que la CEDA había perdido, imponiéndose el candidato del resto y perjudicando los "compromisos no-políticos de la CEDA". ${ }^{8}$

Unos meses más tarde, en setiembre, el conflicto superaba los límites de la institución, con un enfrentamiento entre Bausili (concejal municipal de la Lliga Catalana) y Torrents (consejero-gestor de la CEDA en la Generalitat). ${ }^{69}$ Torrents criticaba la gestión de la Bolsa Municipal de Empleo y denunciaba injerencias en la bolsa de empleo autonómica por parte del ayuntamiento, mientras Bausili, que era el responsable de Política Social, defendía la actuación municipal. Finalmente, el 16 de setiembre, se cesaba el director de la Oficina Local Obrera, Francesc Cañadas Gonzalbo, una derrota de la Lliga enfrente de la CEDA y los radicales.

\section{1. ¿Elecciones municipales?}

En el mismo mes de septiembre, casi sin obra por parte de la comisión gestora, se anunciaban oficialmente elecciones municipales. Pero Cataluña tenía las garantías suspendidas, por lo que, 'La Humanitat', diario portavoz de ERC, consideraba que sin previo acondicionamiento de tiempo y de libertad y garantías absolutas, las izquierdas no podrían ni deberían aceptar las elecciones. ${ }^{70}$

El 7 de septiembre, el ministro de Gobernación, Manuel Portela, insistía ante los periodistas en celebrar las elecciones municipales en el mes de noviembre, con previo restablecimiento de las Garantías Constitucionales y habiendo sido aprobada una ley

\footnotetext{
${ }^{66}$ La Publicitat, 24-7-1935, p. 1.

${ }^{67}$ La Publicitat, 14-7-1935, p. 1.

${ }^{68}$ La Publicitat, 24-7-1935, p. 3.

${ }^{69}$ La Vanguardia 30-8-1935, p. 6. Barcelona. La Vanguardia.

${ }^{70}$ La Humanitat, 7-9-1935, p. 3.
} 
municipal. ${ }^{71}$ Pero sucedió un hecho inesperado. El 3 de noviembre Joan Pich i Pon dejaba el cargo de alcalde por el caso Strauss, ${ }^{72}$ mientras que en la Generalitat de Catalunya se situaba interinamente Eduardo Alonso Alonso, presidente de la Audiencia Territorial de Barcelona. Las elecciones municipales previstas nunca llegarían a celebrarse.

En el mes de setiembre, Alejandro Lerroux, también involucrado en el caso Strauss, era apartado de la presidencia del Consejo de Ministros y era sustituido por Joaquín Chapaprieta, diputado independiente y ministro de Hacienda hasta ese momento, que formaba un gobierno también con presencia de la Lliga Catalana, hecho que sucedía por primera vez desde el inicio de la República. El 29 de octubre eran apartados los ministros radicales, por el caso Nombela, pero sí que se mantenían los ministros de la CEDA y de la Lliga. $^{73}$

A partir de entonces, el equilibrio en el Ayuntamiento de Barcelona, de tres grandes partidos con preponderancia de los radicales, se rompió. El PRR estaba en pleno proceso de desintegración, y la Lliga, dentro del gobierno y con una base política y social mucho más sólida en Barcelona, pasaba de quedar a la sombra del Pacto de Salamanca, entre PRR y la CEDA, a ser la formación mejor situada para conseguir la alcaldía si se modificaba la composición de la comisión gestora. Sin embargo, Jaumar como primer teniente de alcaldía, era temporalmente alcalde accidental interino. ${ }^{74}$

\section{Los radicales pierden el control de su Ayuntamiento}

El presidente de la asamblea municipal, Xavier Calderó (Lliga), en la primera sesión del 14 de noviembre daba cuenta de la marcha del alcalde Pich i Pon, y explicaba que no se podía elegir alcalde porque solo había catorce concejales de elección popular y que el resto no podían elegir a derecho a ley si no tenían la confianza del nuevo gobernador, ya que este podía modificar el ayuntamiento con las designaciones que considerara oportunas.

El concejal radical, también de elección popular, Frederic Frigola, se desmarcaba de su minoría y defendía que se llevaba infringiendo la ley municipal desde el 7 de octubre de 1934 y que se debería de haber formado un consistorio solo con concejales de la Lliga Catalana y de los radicales.

\footnotetext{
${ }^{71}$ La Humanitat, 8-9-1935, p. 7.

72 Ayuntamiento de Barcelona. GM-281/2. AMCB.

73 THOMAS, Hugh: “The Spanish Civil War”., Londres, Penguin Books, 1961 p. 140.

${ }^{74}$ AMCB. Ayuntamiento de Barcelona., GM-281/2.
} 
El nuevo jefe de la minoría radical, Jesús Ulled, le llamaba la atención porque el discurso desautorizaba su jefe político, Pich i Pon, pero Frigola, insistía en que los catorce concejales por derecho propio que estaban presentes sí que podían nombrar un nuevo alcalde. Ya sin ningún tipo de contención hacía su partido, el concejal denunciaba que los gestores no tenían ningún derecho a funciones negativas (como destituir funcionarios), que se habían cometido excesos de todo tipo y que ahora el alcalde tendría que ser un concejal de elección popular. Ulled, designado por Pich i Pon, desautorizaba Frigola y remarcaba que la minoría radical había dado su asentimiento al aplazamiento de elección de alcalde, defendía la forma en que fueron elegidos los ayuntamientos y reconocía que el alcalde accidental interino era Francesc Jaumar.

El concejal Albert de Quintana (CEDA) por su parte defendía que todos los concejales eran gubernativos y se oponía a que hubiera categorías; mientras Soler Janer, como minoría unipersonal daba resignadamente la razón a Frigola, pero le advertía que era inútil dijera lo que dijera, porque no habría alcalde hasta que no hubiera gobernador, porque la decisión final dependía del reparto de puesto que se hacía en Madrid.

La situación de enfrentamiento no terminó. En el mismo pleno se aprobaba un dictamen del concejal regionalista Codolà, responsable de Cultura, para subvencionar escuelas privadas; de nuevo, la oposición la marcaba el radical Frigola, que se oponía a la propuesta. Mientras, la voz oficial de los radicales, Jesús Ulled, con “conllevancia”, decía que se oponía a la enseñanza religiosa y que, por tanto, se comprometía a votar a favor si el consejero municipal Codolà prometía que no se subvencionaría ninguna escuela confesional.

Codolà no respondió y simplemente pidió votación. Fue aprobada por 27 votos a favor y 11 en contra. Los radicales, sin Pich, estaban en clara minoría en el ayuntamiento que ellos mismos habían creado. ${ }^{75}$

\section{La CEDA y la Lliga Catalana al frente del ayuntamiento}

La situación con los radicales en descomposición era de una lucha entre la Lliga y la CEDA por el control del Ayuntamiento. El vicepresidente de la asamblea, Francesc Torres, convocaba un pleno por orden de Jaumar, para la elección del alcalde aprovechando que Calderó, regionalista y presidente de la asamblea, había sido convocado de manera urgente en Madrid y que había sido nombrado Ignasi Villalonga, el cual no había hecho cambios en la composición del ayuntamiento. El nuevo gobernador era de la Derecha Regional Valenciana, integrado en la CEDA, pero que personalmente tenía simpatías por la Lliga. ${ }^{76}$

\footnotetext{
75 La Humanitat, 15-11-1935, p. 8.

76 ARACIL, Rafael: "Memòria de la transició a Espanya i a Catalunya"., Barcelona, Ediciones Universidad de Barcelona, 2000, p. 108.
} 
El alcalde accidental interino se reunía con Ulled, jefe de la minoría radical, ya que, si se sumaban los votos de la CEDA, de los radicales (Frigola se ponía en duda) y los concejales económicos, se tenía mayoría para ser alcalde formalmente. Pero, finalmente, Calderó llegaba a tiempo para firmar la desconvocatoria del pleno y evitar así que Jaumar fuera elegido. En este primer movimiento había "vencido la Lliga", ${ }^{77}$ pero la batalla ya estaba abierta y era pública.

El análisis que se hacía, era que, con seis concejales de elección gestora, los radicales tenían unos intereses más cercanos a la CEDA, pero sin incitar a que la ruptura con la Lliga Catalana llevara a elecciones, que expulsaría a los partidos sin votos del consistorio. 'La Publicitat' ya señalaba a Coll i Rodés como posible alcaldable de la Lliga. ${ }^{78}$

Los primeros días de diciembre se produce un nuevo episodio, que supone la marcha definitiva de Frigola de los radicales. En sesión plenaria se presentaba el Contrato de Tesorería y Andreu Bausili señalaba que el cupón vencía el 31 de diciembre y que no había nada previsto para pagarlo. El radical Matheu se quejaba de que no habían tenido tiempo para estudiarlo y acusaba a los financieros de la comisión de ejercer una dictadura en el ramo, pidiendo un aplazamiento de diez días. Jaumar decidía suspender la sesión solo dos días, pero Frigola se quejaba de que se había pedido la urgencia del dictamen y sin dejar hablar sobre la urgencia se suspendía la sesión, "lo que daba a la urgencia un vicio de nulidad". 79

Posteriormente, el agrario Viza, responsable de Finanzas, defendía el dictamen de las bases del contrato de tesorería, pero consideraba que debería ser el propio ayuntamiento el que prestara el servicio, ya que tenía suficientes técnicos y se evitaban de pagar otra vez el millón de pesetas en comisiones que habían desembolsado en el contracto que caducaba. Los radicales decían que, si la mayoría votaba las bases, ellos votarían a favor, mientras Francesc Torras anunciaba el voto en contra, acusando a los concejales de la CEDA y la Lliga de hacer una maniobra y pedía aplazar la votación hasta el 31 de diciembre si esta era la fecha límite. Frigola por su parte decía que votaría en contra, y Ulled le señalaba que con su acto se había apartado de la minoría radical voluntariamente. La respuesta: "es un honor el separarse de la minoría radical y hasta sería un honor ser expulsado". El dictamen quedaba aprobado. ${ }^{80}$

La política estatal estaba en crisis y, aprovechando la inestabilidad, se convocaba un pleno para el 10 de diciembre para la elección de alcalde. Sin embargo, finalmente los números no salían. Los nueve votos de la CEDA, más el mequiadista Roviralta, sumaban diez; y en frente había nueve de la Lliga, más el tradicionalista Soler Gener, también diez. La

\footnotetext{
${ }_{77}$ La Publicitat, 24-11-1935, p. 3.

${ }^{78}$ La Publicitat, 27-11-1935, p. 3.

${ }^{79}$ La Humanitat, 3-12-1935, p. 8.

${ }^{80}$ La Humanitat, 5-12-1935, p. 8.
} 
abstención de radicales (ocho), técnicos (seis), de los agrarios de Viza y de Frigola, mostraba una situación de empate entre las partes, insuficiente para que saliera elegido cualquiera de los candidatos.

El aplazamiento era visto esta vez como una victoria para Jaumar que, como alcalde gestor interino, seguía al frente del ayuntamiento. El pleno se aplazaba con los votos en contra de la Lliga en el Consejo de Gobierno. ${ }^{81}$

\section{La marcha de la CEDA y el gobierno de la Lliga Catalana}

El 14 de diciembre se formaba en el Estado un gobierno del centrista Manuel Portela, anunciando que disolvería las Cortes. El gobierno sin apoyo de las derechas, y, por tanto, sin la confianza necesaria del Parlamento, provocaba que, para evitar la derrota, Alcalá-Zamora suspendiera las sesiones hasta el primer día de 1936.

En esta situación dimitía Ignasi Villalonga, después de solo veinte días en el cargo, siendo sustituido interinamente por Joan Maluquer i Viladot, de la Lliga Catalana. Partido que sí que formaba parte del nuevo gobierno, con Pere Rahola como ministro sin cartera.

El 15 de diciembre, ya sin la confianza del gobernador, la CEDA abandonaba el ayuntamiento y la Generalitat, y el ya ex alcalde Francesc Jaumar declaraba que la solución de la crisis se debía "a las ignominiosas intrigas de la Lliga" ${ }^{82}$ El nuevo alcalde accidental sería el hasta ese momento primer teniente de alcalde, el lerrouxista Jesús Ulled, ${ }^{83}$ también de designación gubernativa y que afirmaba en nombre de los radicales que "si nosotros no dimitimos fue porque el señor Lerroux nos ordenó que no lo biciéramos, porque este es un Ayuntamiento de colaboración de partidos y no de lucha".

La Lliga ya sin la rivalidad de la CEDA, hablaba de un pleno para elegir un alcalde. Además, a la situación se añadía el nombramiento el día 18 como gobernador general de Fèlix Escalas i Chamení, político independiente vinculado a la Lliga ${ }^{84}$ Escalas nombraba las vacantes de consejeros municipales en substitución del radical Joan Pich i Pon, y de los cedistas, con los candidatos no elegidos de la Lliga Catalana en las elecciones municipales de 1934: Josep Maria Cardona i Espuñez, Montserrat Serra i Bellsolell, Frederic Amat i Arnau, Jaume Espona i Brunet, Francesc Guarner i Molins, Manuel Baster i Duran, Alfons d'Oriola i Cortada i Renom, Josep Maria Ginestar i Ponç, Alfred Dorca i Blanc y Joan Andreu i Miralles. ${ }^{85}$

${ }^{81}$ La Publicitat, 11-12-1935, p. 3.

82 La Publicitat, 17-12-1935, p. 1.

83 AMCB. Ayuntamiento de Barcelona. GM-56. Circulares Varias.

${ }^{84}$ La Publicitat, $20-12-1935$, p. 3.

${ }^{85}$ La Publicitat, 21-12-1935, p. 3. 
El gobernador Escalas justificaba el nombramiento con solo concejales de la Lliga Catalana porque ERC no quería formar parte y los radicales ya contaban con representantes no elegidos por sufragio y con un número muy superior según el peso electoral. ${ }^{86}$

Los diez nuevos concejales, entre los que estaba el carlista Francesc Guaner, daban la mayoría absoluta la candidatura de la Lliga Catalana y de los tradicionalistas. Cabe mencionar la renuncia de Francesca Bonnemaison de formar parte de la comisión gestora, pero en cambio sí que formaba parte Montserrat Serra i Bellsolell, de Roig-Chova, la primera concejala del Ayuntamiento de Barcelona durante el período republicano.

Ulled quería dejar la vara inmediatamente, indignados los radicales porque incluso el ex alcalde Pich i Pon había sido sustituido por un regionalista. Para la Lliga esto supondría un descalabro porque hacía inútil la celebración de un pleno, o sea, la elección 'democrática' de un nuevo alcalde. Finalmente Ulled cedía y se esperaba a la elección.

El 24 de diciembre se celebraba la sesión para elegir alcalde, ${ }^{87}$ en la que era elegido Ramon Coll i Rodés con 25 votos a favor y 14 en blanco. Coll i Rodés había sido del Partit Republicà Català hasta enero de 1933 y se intentaba dar una imagen más catalanista y menos conservadora de cara a las siguientes elecciones.

La Comisión de Gobierno estaba formada por los siguientes consejeros-concejales: ${ }^{88}$

\section{Cuadro 4}

\section{Lista de la última Comisión de Gobierno Municipal durante los gobiernos gestores}

\begin{tabular}{|l|l|l|}
\hline Nombre & Área & Partido político \\
\hline Ramon Coll i Rodés & Presidente & Lliga Catalana \\
\hline Jesús Ulled & Ensanche & Partido Republicano Radical \\
\hline Josep Codolà & Cultura & Lliga Catalana \\
\hline Andreu Bausili & Fomento & Lliga Catalana \\
\hline Josep Farrero & Circulación & Partido Republicano Radical \\
\hline Ferran de Segarra & Política Social & Lliga Catalana \\
\hline Joan Baptista Viza & Finanzas & Partido Agrario Español \\
\hline Frederic Roda i Ventura & Gobernación & Lliga Catalana \\
\hline Frederic Amat & Provisiones & Lliga Catalana \\
\hline
\end{tabular}

Fuente: Elaboración propia

\footnotetext{
${ }^{86}$ La Publicitat, 24-12-1935, p. 3.

${ }^{87}$ La Publicitat, 22-12-1935, p. 3.

${ }^{88}$ La Publicitat, 29-12-1935, p. 4.
} 
En la última y única sesión en la cual Coll i Rodés actuó como alcalde, se intentó marcar un perfil propio, aprobando un presupuesto de obras por valor de 21,8 millones de pesetas, de los cuales quince debían ser adquiridos mediante la emisión de títulos. 'La Humanitat' la bautizó como "sesión de testamento". 89

\section{Consecuencias y conclusiones}

La suspensión de los concejales elegidos en las elecciones municipales de 1934 era generalizada, ya que más del setenta por ciento de la población catalana estaba gobernada por una comisión gestora y no había ningún municipio de más de diez mil habitantes que no tuviera el ayuntamiento popular suspendido. ${ }^{90}$

El 7 de enero se disolvían las Cortes, se restablecían los derechos constitucionales y se reabrían los centros clausurados. Las izquierdas catalanas habían sido apartadas de las instituciones, y sus principales dirigentes, como participantes en la proclamación del Estado catalán, encarcelados y condenados a largas penas. ${ }^{91}$ Este escenario facilitó que se unieran todas estas formaciones en un Front d'Esquerres (Frente de Izquierdas) de cara a las elecciones generales, las cuales eran convocadas para el 16 de febrero de $1936 .{ }^{92}$

Los elementos principales del programa eran la amnistía, la restauración de autonomía, el restablecimiento de los ayuntamientos electos, así como mantener los progresos sociales y evitar una revisión conservadora de la Constitución. ${ }^{93}$ Con unas confluencias similares a nivel estatal, con el Frente Popular, las elecciones fueron totalmente polarizadas entre las izquierdas y los partidos de derechas, que habían gobernado durante este periodo transitorio.

En un sistema mayoritario como el de la Segunda República, se perjudicaba la división, ${ }^{94}$ y la reacción de las derechas fue hacer también, pese a las diferencias, una lista conjunta. En el caso de Cataluña, la candidatura se denominó Front Català d'Ordre (Frente Catalán de Orden). La Lliga Catalana era el partido con más candidatos, con 20 de los 54 que

\footnotetext{
${ }^{89}$ La Humanitat, 28-1-1936, p. 8.

90 La Humanitat, 23-11-1935, p. 1.

91 POBLET, Josep Maria: “Història de l'Esquerra Republicana de Catalunya. 1931-1936”., Barcelona, Dopesa, 1976 , p. 247.

92 TUNÓN DE LARA, Manuel: 'La crisis de Estado: dictadura, república, guerra (1923-1939)”., Barcelona, Labor, 1981, p. 212.

93 La Publicitat, 21-1-1936, p. 1.

${ }^{94}$ MONTERO, José R.; LLERA, Francisco J.; TORCAL, Mariano: “Sistema electorales en España: una recapitulación”., Reis. Revista Española de Investigaciones Sociológicas, no 58 (1992), p. 7-56. p.12.
} 
habían en Cataluña; la segunda formación era Acció Popular Catalana (adherido en la CEDA), con 9, los tradicionalistas tenían 5, el PRR 4, y un candidato o dos para el resto de formaciones. $^{95}$

No había un programa común y tampoco se podía apelar a una obra de gobierno a nivel municipal. La actuación se había caracterizado por los enfrentamientos entre los socios y unos resultados escasos y de carácter técnico. Cabe remarcar que los cambios hechos, como los nombramientos, ascensos o los nuevos contratos con las compañías de autobuses, fueron posteriormente anulados una vez restituidos los ayuntamientos surgidos en las elecciones municipales de 1934.

Por lo tanto, en términos generales, el periodo gestor no sirvió ni para consolidar a los radicales ni al bloque de partidos de centroderecha en conjunto; en cambio, sí que unió a las izquierdas y focalizó el debate en la amnistía, los progresos sociales y el autogobierno, cuestiones que tenían un enorme apoyo popular.

El periodo de los gobiernos gestores no sirvió de presentación de un modelo alternativo, sino que se impuso el enfrentamiento entre los socios de comisión gestora. Lo cierto es que las derechas nunca pudieron gobernar ninguna institución catalana duranta la República a través de las urnas.

La Lliga utilizó principalmente dos argumentos en campaña. El primero era un discurso del miedo, remarcando que los socialistas serían los que se impondrían a los republicanos, porque tenían la mayoría del voto de izquierdas en España y se iría a una situación similar a la del 6 de octubre. El otro argumento, más interesante, era que, si las izquierdas se imponían en Cataluña y las derechas ganaban en el resto de España, no habría Estatuto y se mantendría el régimen transitorio que había en ese momento. En cambio, Joan Ventosa i Cavell, el cabeza de lista, afirmaba que, pese que había “bombres anticatalanistas", si ganaban, estos tendrían que reconocer que se "ban triunfado gracias al voto de los catalanistas" y "entonces se rendirán a la evidencia y serán más benévolos con el catalanismo". Por lo tanto, según reivindicaban, la Lliga Catalana y la candidatura de derechas eran la garantía que, mandara quien mandara en Madrid, se restablecería el Estatuto y la autonomía. ${ }^{96}$

En las elecciones del 16 de febrero de 1936, el Frente Popular conseguía una mayoría absoluta de escaños en España y, en la ciudad de Barcelona, la candidatura del Front d'Esquerres lograba una clara victoria, con un porcentaje de voto superior al 60\%, y dejando a Cambó (Lliga), Lerroux (PRR) y Cirera i Voltà (CEDA) sin acta en Cataluña. ${ }^{97}$

95 MOLAS, Isidre: "El sistema de partidos políticos en Cataluña. 1931-1936”, Barcelona, Ediciones Península, 1973, p. 169.

${ }^{96}$ La Humanitat, 26-1-1936, p. 1.

${ }_{97}$ TARRAGONA, Eduardo: “Las elecciones de 1936 en Cataluña”., Barcelona, Bruguera, 1977, p. 240. 
Un día después de la votación, Carles Pi Sunyer iba a ocupar la alcaldía, ${ }^{98}$ aunque no sería hasta el 18 cuando se restablecerían oficialmente los ayuntamientos populares.

Como explica Carles Pi Sunyer, tras la victoria electoral, se producían concentraciones en la Plaza de la República [actual Plaza de Sant Jaume], con la gente pidiendo un acto de fuerza. Como actuar contra la Generalitat supuso que podía ser peligroso, decidió que era mejor el ayuntamiento para dar salida a las reclamaciones. ${ }^{99}$ Para llevar a cabo la acción y recuperar el poder en el consistorio, Pi Sunyer llamó a Félix Escalas, que consideró correcta la acción, y a Ramon Coll i Rodés, que Pi Sunyer consideraba y que incluso se mostró "encantado y aliviado". ${ }^{100}$ Los gobiernos gestores llegaban así a su fin.

\footnotetext{
${ }^{8}$ Gaceta Municipal de Barcelona (1914-2020). Ayuntamiento de Barcelona. Servicio de Documentación y Acceso al Conocimiento (SEDAC), 1936, n. 8 al 11.

${ }^{9}$ PI SUNYER, Carles: op. cit., p. 353.

100 PI SUNYER,Carles: op. cit. p. 354.
} 